\title{
Automated Multi-Scale Microstructure Heterogeneity Analysis of Selective Electron Beam Melted TiAl6V4 Components
}

DOI:

10.1002/9781119093466.ch54

\section{Document Version}

Accepted author manuscript

Link to publication record in Manchester Research Explorer

\section{Citation for published version (APA):}

Prangnell, P., Zhao, H., Antonysamy, A. A., Meyer, J., \& Williams, S. W. (2015). Automated Multi-Scale Microstructure Heterogeneity Analysis of Selective Electron Beam Melted TiAl6V4 Components: english. 429. Paper presented at TMS 2015 A146th Annual Meeting and Exhibition, Orlando, United States. https://doi.org/10.1002/9781119093466.ch54

\section{Citing this paper}

Please note that where the full-text provided on Manchester Research Explorer is the Author Accepted Manuscript or Proof version this may differ from the final Published version. If citing, it is advised that you check and use the publisher's definitive version.

\section{General rights}

Copyright and moral rights for the publications made accessible in the Research Explorer are retained by the authors and/or other copyright owners and it is a condition of accessing publications that users recognise and abide by the legal requirements associated with these rights.

\section{Takedown policy}

If you believe that this document breaches copyright please refer to the University of Manchester's Takedown Procedures [http://man.ac.uk/04Y6Bo] or contact uml.scholarlycommunications@manchester.ac.uk providing relevant details, so we can investigate your claim.

\section{OPEN ACCESS}




\title{
Automated Multi-Scale Microstructure Heterogeneity Analysis of Selective Electron Beam Melted TiAl6V4 Components
}

\author{
H Zhao $^{1}$, A. A. Antonysamy ${ }^{2}$, J. Meyer ${ }^{3}$, O. Ciuca ${ }^{1}$, S. W. Williams ${ }^{1}$, P. B. Prangnel ${ }^{1}$ \\ ${ }^{1}$ Materials Science Centre, University of Manchester, Grosvenor St. Manchester M13 9PL, UK \\ ${ }^{2}$ GKN Aerospace, GP. O. Box 500, Filton, BS34 9AU, UK \\ ${ }^{3}$ Airbus Group Innovations, Golf Course Lane, Filton, Bristol, BS99 7AR, UK \\ Keywords: "Additive manufacturing, SEBM, Ti-6Al-4V, Microstructure"
}

\begin{abstract}
In additive manufacturing (AM) the moving heat source and layer deposition gives rise to each volume of material receiving a complex thermal history. In addition, the machine control systems can vary the heat input as a function of the component geometry and process themes. Together, this can potentially cause both short and long range microstructure heterogeneity, which can potentially impact on the local mechanical properties of AM components. To systematically quantify the heterogeneity typically seen in the lamellar microstructures found in AM titanium parts, a tool has been developed that combine's automatic high resolution SEM image mapping with batch image analysis, to enable efficient quantification over large areas at the required resolution. This method has been applied to parts produced in Ti6Al4V by selective electron beam melting using an Arcam machine. The method and test cases are described, where both long and short range heterogeneity have been identified in samples and correlated to the build parameters.
\end{abstract}

\section{Introduction}

Additive manufacturing (AM) is a novel technology that can build 3D components directly from CAD files. Unlike traditional subtractive processes, which are mainly suited for mass production, AM favors small production runs of complex components, with expensive materials, as it requires minimal tooling costs, and results in low material waste [1]. The application of additive manufacturing to Ti-6Al-4V parts in aerospace has received a lot of attention over the past decade $[2,3]$. To date, the potential of several AM processes, such as direct metal deposition (DMD) [4], selective laser melting (SLM) $[1,5,6]$ and selective electron beam melting (SEBM) [7], have been extensively investigated for producing safety-critical aerospace components. For such applications the SEBM process has certain advantages over SLM, in terms of a lower defect density, lower residual stresses, and larger part dimensions, while preserving a high geometric flexibility $[1,7]$. In SEBM with the Ti-6Al-4V alloy the final microstructure, after cooling down, is generally comprised of a mixture of fine colony and Widmanstätten lamellar $\alpha$, with a small volume fraction of retained $\beta$ on the $\alpha$ plate boundaries [7].

In additive manufacturing (AM) the moving heat source and layer deposition results in each volume of material receiving a complex thermal history. For example, as each track is melted by the heat source the material underneath in the previous layer will be reheated with a rapid thermal cycle in a similar manner to that seen in a weld HAZ. Generally this occurs several times with a diminishing peak temperature [4]. As a result, microstructural banding can often be seen in the transformation structure of alloys like Ti-6Al-4V [2, 4]. In addition, AM machines use control algorithms to ensure a consistent melt pool depth and also to try to maintain a constant 
temperature with build height. This can, for example, involve speeding up the beam when reversing the track path in hatching routines, which locally reduces the energy input per unit volume [6]. Powder bed AM systems also generally draw the outline of a section in each layer using different beam parameters to when they infill by hatching. [6] It has previously been shown that this can lead to different primary $\beta$ grain structures, as a function of wall thickness [8], but will also result in local differences in energy density near a section edge and it is likely that this could affect the $\alpha+\beta$ transformation structure in titanium components. With greater part geometric complexity, it is thus likely that microstructural heterogeneity will become more pronounced due to the increasingly complicated thermal conditions. If significant microstructural heterogeneity is present within AM components, clearly this can affect the local mechanical properties, which would make the qualification of aerospace components more difficult. However, currently few attempts have been made to quantify the level of microstructure variation produced by different AM platforms, or relate this to specific geometry and control issues when building complex components.

The present study has thus attempted to assess the level of microstructural heterogeneity found in SEBM AM components by using an automated multi-scale approach. This has involved combining high resolution SEM imaging with automated mapping across large areas, followed by batch image processing, based on a Matlab program. The technique has been applied to quantify the dimensions of the $\alpha-\beta$ lamellar microstructures seen in typical AM components produced in Ti-6Al-4V using two example parts; an early build demonstrator hinge, built with non-optimized parameters, and a simple very tall square section test sample.

\section{Experimental Methods}

\section{Material Production}

The specimens analysed were produced using an 'Arcam' A2 machine (Fig. 1) at Airbus and GKN's additive manufacturing centre, UK. The Arcam EBSM machine uses a $3.5 \mathrm{~kW}$ thermionic tungsten filament e-beam gun and a vacuum of about $10^{-7}$ mbar. The powder feedstock was grade $5 \mathrm{Ti}-6 \mathrm{Al}-4 \mathrm{~V}$, with a particle size range from $45-110 \mu \mathrm{m}$ in diameter (supplied by Arcam Sweden). The scanning patterns used were the standard machine setting 'contour \& hatching' schemes, where the e-beam is focused to $0.2-0.3 \mathrm{~mm}$ in diameter. The layer increment height was $50 \mu \mathrm{m}$. Before depositing each layer, the powder bed was pre-heated by the beam to a target temperature of $750{ }^{\circ} \mathrm{C}$.

Two specimens were used for the case-studies presented in this paper: 1) a web region cut from a complex shaped hinge part (Fig. 1(b-c)); 2) a very tall cubic cross-section bar that was built to the maximum height of the build chamber $(\sim 30 \mathrm{~cm})$ (see Fig. 1(d)).

\section{Sample Preparation and SEM Mapping}

Specimen cross-sections were first prepared by mechanical grinding and then polishing to $1 \mu \mathrm{m}$ diamond, with a final gentle colloidal silica suspension polish. After etching with Kroll's reagent for 10 seconds, optical microscopy was used to locate features of interest. The samples were then re-polished and mapped in an FEI high resolution Magellan SEM, using 'MAPS' software which can automatically takes sequential images at predetermined positions. These can be adjacent tiles, to fully map a small region, or regularly stepped to allow systematic sampling over large areas. A fixed image field width of $50 \mu \mathrm{m}$ was selected for the individual images so as to adequately resolve the fine $\alpha-\beta$ microstrucurre in the SEBM materials (see Fig. 2), except for the banding structure analysis (see below) where a smaller value of $6.35 \mu \mathrm{m}$ was used to resolve variation at a smaller length-scale. 

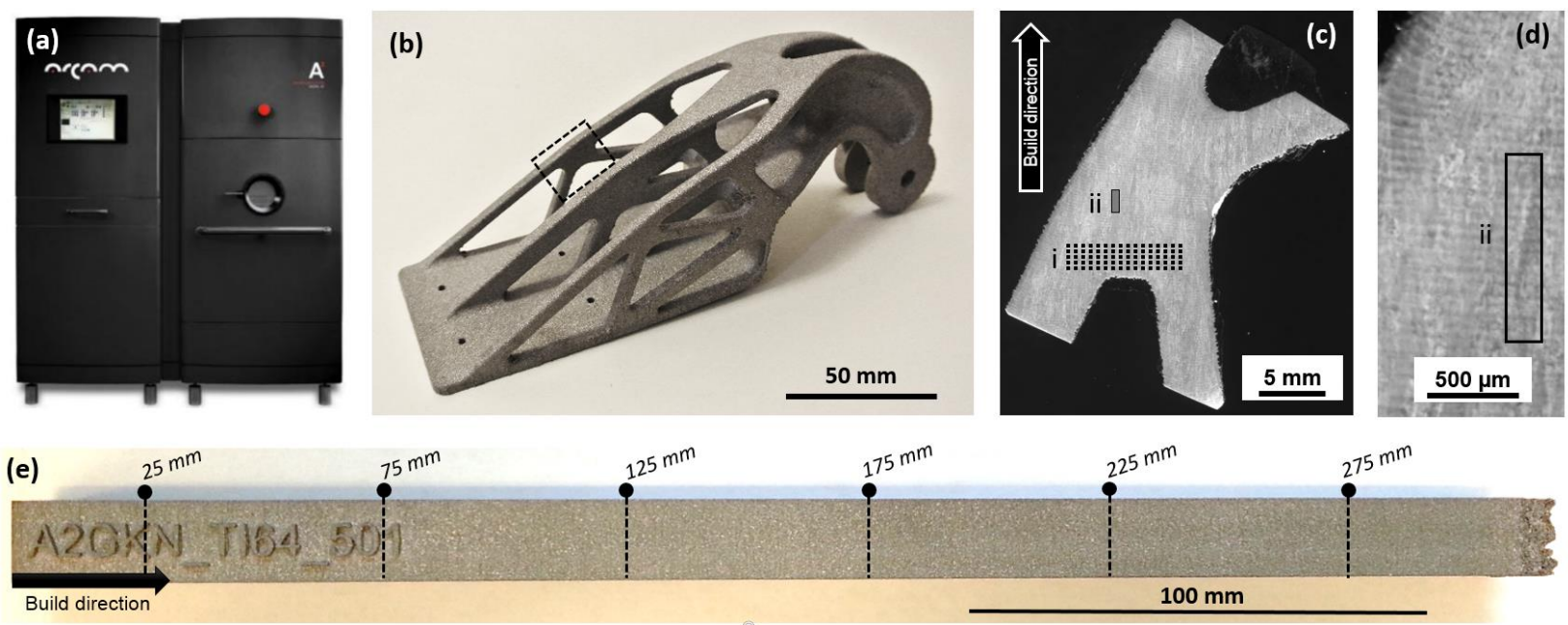

Fig. 1. (a) The Arcam A2 SEBM machine used in this study; (b) a demonstrator hinge part used for case study 1; (c) the K-shaped web specimen cut from the hinge showing schematically the areas analysed; i) a corner over hang area \& ii) a central region across several layers; (d) the banded structure and region ii) at high magnification; (e) the square cross section bar used for case study 2 , built to the maximum chamber height, with the sampling positons indicated.

In the hinge part, stitched image tile maps were produced to study important regions at high resolution. An overhang area was selected to be mapped where a 'lack of fusion' defect was known to be present in this early development sample (area i. in Fig. 1(c)). For this region a $5 * 32$ image matrix was taken across a rectangle of $200 \mu \mathrm{m}$ by $6 \mathrm{~mm}$ 's. A second set of images was taken from the central region to see if any banding could be detected across the layers, using a

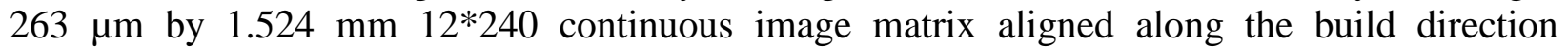
(perpendicular to the layers). A reduced window size, i.e., $6.35 \mu \mathrm{m}$ in the build direction and 22 $\mu \mathrm{m}$ in transverse direction, was used in this case to resolve the fine layer height.

In contrast, the long square-section bar was analysed over a larger length scale to evaluate any long range heterogeneity that might be present across the bar and along its length. The bar was first sliced at 6 different heights (shown in Fig. 1(e)). The slices were then mounted and polished together to obtain optimal imaging consistency. $6 * 60$ image arrays were then taken at the same magnification across the slices with an incremental spacing of $0.25 \mathrm{~mm}$.
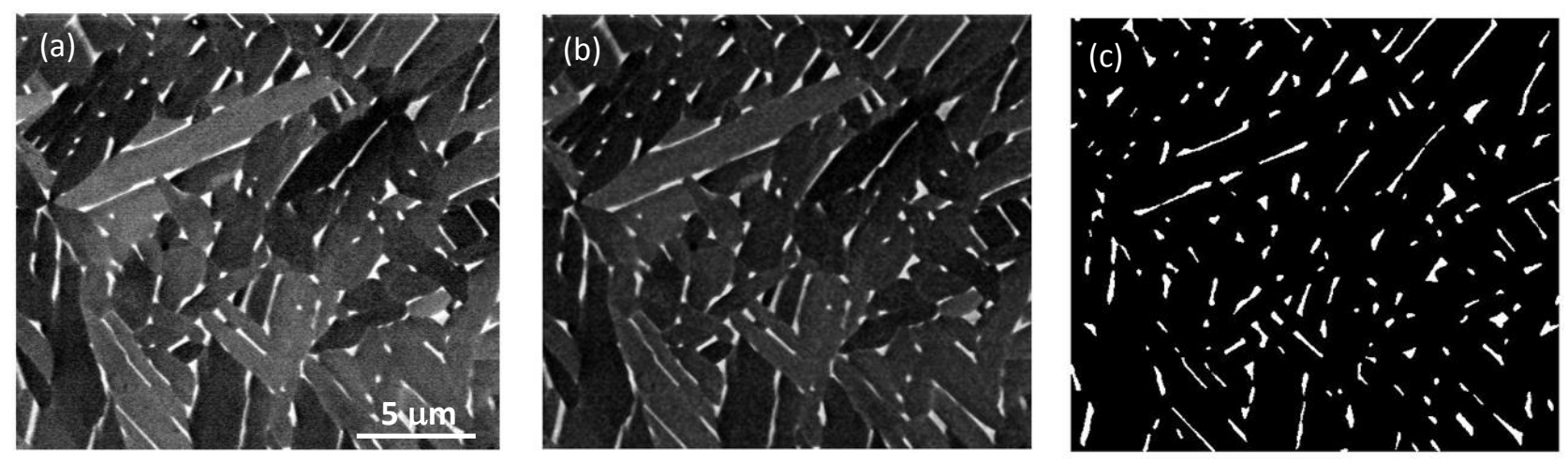

Fig. 2. (a) Original SEM image with $Z$ contrast; (b) optimized image: after dynamic background filtering, image enhancement, and image smoothing; (c) a final binarized image: dark $=\alpha$ phase, bright $=\beta$ phase. 


\section{Image Analysis}

When using the FEI MAPS software to create image maps the focus is automatically adjusted and all the operating conditions are kept as constant as possible. However, small changes in the working distance, electron beam stability, surface contamination etc., can change the image brightness and contrast. Back scaterted images were used to ensure strong differntiation between the $\alpha$ and $\beta$ phases and their contrast is also strongly affected by the local crystal orientation. To adjust for these effects, so that consistent results could be obtained, automated image enhancement was first applied to each image (e.g. Fig. 2) to adjust them for brightness and contrast consistency $[9,10]$. In addition, an 'adaptive background filter' was used to minimize the contrast between different regions in the same image while preserving the contrast between phases (Fig. 2b). The filter size was determined by comparing the average thicknesses of $\alpha$ and $\beta$ layers. Depending on the features of interest, two methods were then used; differential image processing and binary image processing, respectively. The former method was employed to detect 'edges' or grayscale gradient peaks in the images, and thus effectively detect the phase boundaries between the $\alpha$ and $\beta$ phases. This subsequently allowed the linear intercept method to be applied to calculate the average spacing of the phase boundaries. When separated by a continuous $\beta$ layer, the mean 2D $\alpha$ plate spacing could then be calculated using the following function,

$$
d=\frac{L}{N_{d}}=L /\left(\sqrt{{\overline{N_{x}}}^{2}+{\overline{N_{y}}}^{2}}\right)
$$

where $\overline{N_{x}}$ and $\overline{N_{y}}$ are mean intercepts in x direction and y direction respectively.

In addition, the $\beta$ phase was isolated as segmented particles using a binary filter (Fig. 2c). This allowed the size and mean aspect ratio of the $\beta$ regions to be calculated to evaluate the level of $\beta$ spheroidisation upon prolonged exposure in the SEBM powder bed.

\section{Results and discussion}

\section{Microstructure Variation in an Overhang Structure}

In the ' $\mathrm{K}$ ' shaped web specimen, taken from the AM development sample, used to produce the hinge bracket (Fig.1b), a high pore density was found in both the top and bottom corners, but the density was greatest near the section base, where the corner was built upwards above the powder bed (Fig. 3a). Apart from the presence of spherical gas pores (Fig. 3.b) a high aspect ratio cavities were also observed at this location (Fig. 3.c). These irregular defects have been previously referred to as 'lack of fusion' flaws that are formed due to an insufficient heat input, or overlap between melt tracks $[2,3,6]$.

In the microstructures analysed in the SEBM samples provided (e.g. Fig. 2) it is apparent that the $\beta$ phase does not form a continuous layer between the $\alpha$ plates. This is probably because it has been partially spheroidised by the long time spent at the high build temperature employed $\left(750{ }^{\circ} \mathrm{C}\right)$. This means, in this case, that inferring absolute measurements of $\alpha$ plate width from the phase edge contrast, by using the linear intercept method, is unreliable because the intercept line may pass though $\alpha$ plate boundaries where there is a gap in the $\beta$ phase layer. 

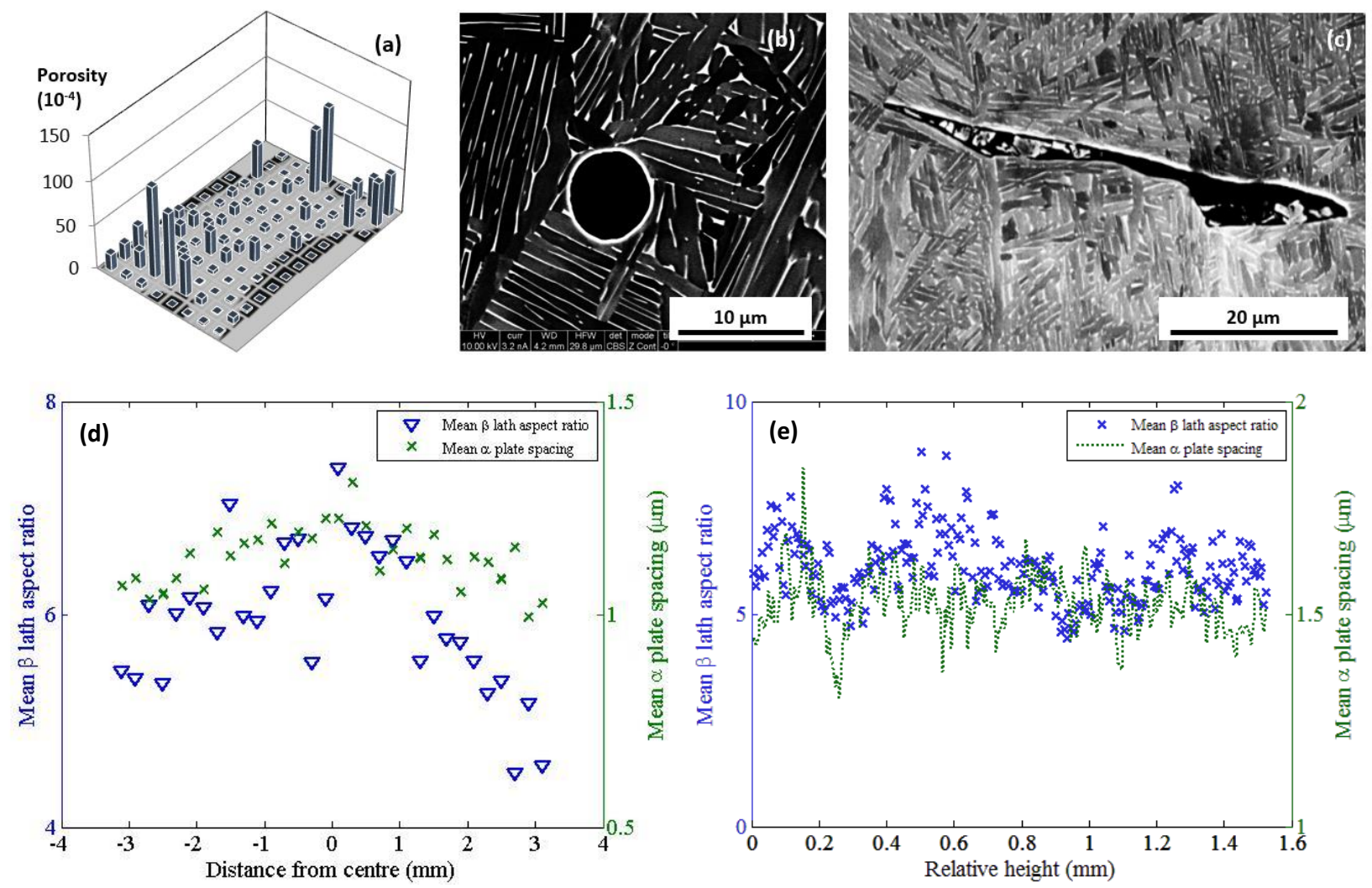

Fig.3. Analysis of selected regions in the K-shaped example hinge specimen (a) spatial distribution of the porosity volume fraction; (b) \& (c) examples of spherical pores and high aspect ratio 'lack of fusion' defects; (d) the phase boundary spacing and mean $\beta$ phase aspect ratio plotted versus horizontal position across the bottom overhang corner (region (i) in Fig 1c), (e) the phase boundary spacing and mean $\beta$ aspect in the centre of the section (region (ii) in Fig 1c) perpendicular to the build direction.

In contrast, the $\beta$ phase aspect ratio is still an accurate direct measurement of the $\beta$ phase morphology. Nevertheless, taken together, statistical measurements of the image map from region (i) of the ' $\mathrm{K}$ ' section (see Fig. 1c), from across the bottom corner overhang, indicates that there $\alpha$ plate widths are nearly uniform across the corner, whereas a much higher $\beta$ phase aspect ratio, or lower level of spheroidisation, was detected where the majority of the defects were located. This microstructure data is therefore consistent with a lower local energy density being applied by the Arcam machine, which has resulted in a less spheroidised microstructure, as well as a greater density of porosity in this region.

In the low magnification optical image in Fig. 2(e) from the centre of the ' $\mathrm{K}$ ' section (location (ii) Fig. 1(d)) evidence of weak microstructural banding can be observed across individual layers. Statistical measurements were therefore performed across this area by analysis of a stitched image map, covering approximately 17 layers of the build. Interestingly, this data showed insignificant long range variation in the phase boundary liner intercept spacing or $\beta$ phase aspect ratio, which indicates that on a fine scale in the centre of the hatched region the microstructure was very homogenous. However, the phase boundary linear intercept data can be seen to be noisy with a regular periodicity. When processed using a Fourier transform, this gave a mean frequency of $57 \mu \mathrm{m}$ which is quite close to the expected layer height. 
Microstructure Variation across a Section Width and with Build Height

In Fig. 4(a) the phase boundary liner intercept spacing and $\beta$ phase aspect ratio have been plotted across a typical section width at half the build height of the tall square section bar. This data was obtained by generating an array of 60 images across the entire section centre line with a regular spacing of $238 \mu \mathrm{m}$. In addition in Fig. 4(b) the same measurements, averaged over several sections, have been plotted as a function of build height. It should be noted that the sample used was built close to the full height of the machine chamber and was stopped at $29.7 \mathrm{~cm}$. From these results, it is very obvious that the microstructure varied significantly both across the sample section and with height, but the variation was much greater in terms of the $\beta$ aspect ratio than the nominal $\alpha$ plate spacing. In Fig. 4(a) it is apparent that the microstructure is more spheroidised within the contour pass region near the sample edge than in the centre hatching region. Interestingly, this observation can be directly linked to the operation of the Arcam machine, which uses a slower speed and higher energy density when contouring, compared to hatching [11]. Furthermore, when hatching a turning function is employed that accelerates the beam when it reverses its path at the end of each hatch line. This function is designed to reduce the heat input to compensate for when the beam path reverses back over material that was recently heated by the forwarded motion of the beam. However, the results here suggest that this function overcompensates and net local energy density was slightly lower at the edge of the hatch area, relative to the part centre.
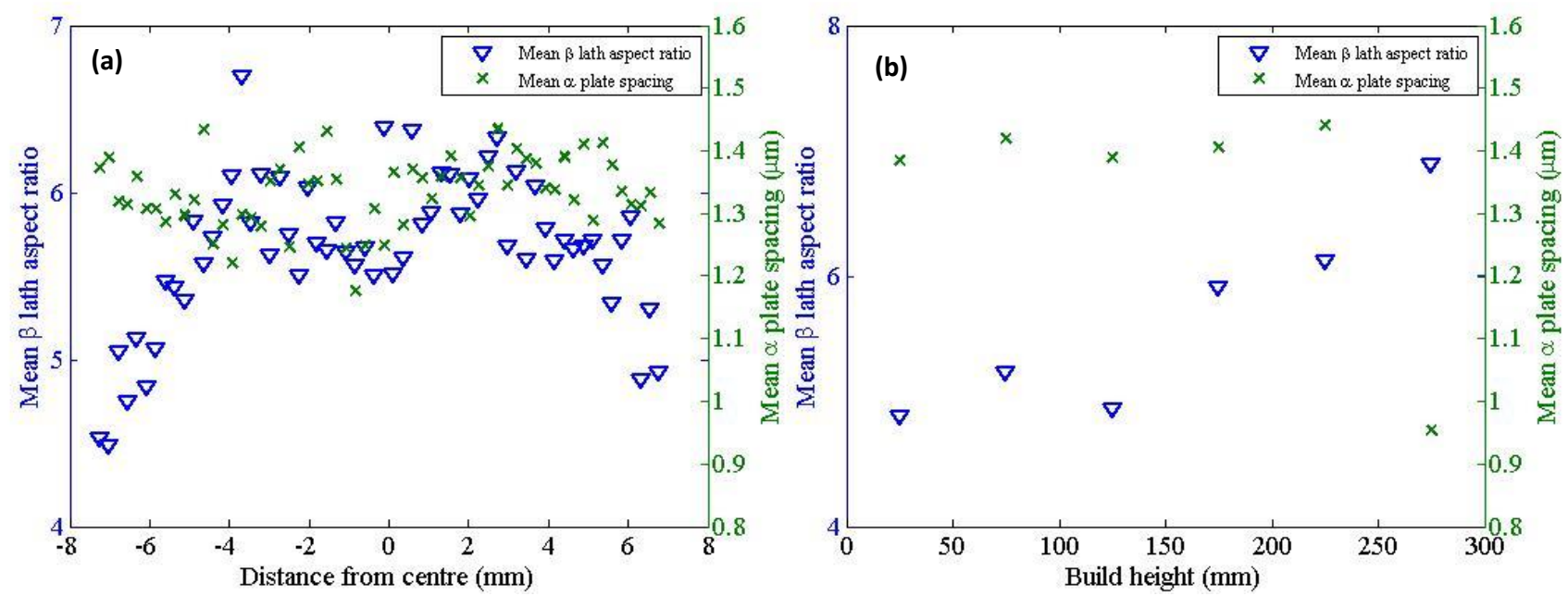

Fig. 4. Phase boundary liner intercept spacing and $\beta$ phase aspect ratio data from the square cross section bar (a) mapped across the full section width and from bottom to top with build height.

In the final example, Fig. 4(b) reveals that in the tall bar sample the average $\beta$ phase aspect ratio increases greatly with build height, with the top to bottom difference in both cases being of the order of $30 \%$, whereas the nominal $\alpha$ plate spacing remained relatively constant. This behaviour probably reflects the difference in time that a piece of material is at temperature when a part is being built, as to achieve this height the build time was overt 48 hours. Thus, the material near the base was at temperature for far longer than that in the last layers deposited at the top of the bar.

\section{Challenges and Issues}

In additive manufactured Ti-6Al-4V, the colony and Widmanstätten lameallar microstructures present naturally generate considerable scatter in quantitative image analysis measurements. This is because of the effect of sectioning a 3D microstructure, in which there is alignment of the $\alpha$ 
lathes within different regions that are dominated by a particular subset of $\alpha$ orientation variants. Therefore, to obtain a valid data point it has been observed that an average of at least 5 images is required. As has been mentioned in the experimental section, when mapping large sample areas quantification can also be sensitive to changes in the imaging condition to a point where this can no longer be corrected for by image enhancement. This has been found to particularly be a problem near the edge of samples where curvature to the surface can be generated during polishing. An automated step for quality assessment is therefore currently being developed, which will allow unreliable data to be discarded from regions of image maps where the results are too greatly affected by image quality. A further problem with the current approach is that we cannot measure a true $\alpha$ plate spacing in a structure where the $\beta$ phase is no longer continuous along the $\alpha$ plate boundaries owing to spheroidisation. This is because with back scatter imaging phase boundary recognition is dependent on the contrast between the $\alpha$ and $\beta$ phases caused by their different compositions. Indeed, when the $\beta$ phase is more broken up the phase boundary linear intercept will result in a larger than actual $\alpha$ plate spacing. However, overall the results show that the $\alpha$ plate width was relatively homogenous with location, whereas the $\beta$ phase aspect ratio varied significantly. This suggests that the $\beta \rightarrow \alpha+\beta$ transformation takes place at a fairly consistent cooling rate throughout a component, but the local residual heat and time at temperature then affects the morphology of the $\beta$ phase.

\section{Conclusion}

An automated microstructure quantification technique has been developed specifically for mapping heterogeneity in Ti6Al4V AM components. This has involved applying dynamic background and contrast correction to tiles of high magnification SEM images over large sample areas. Two microstructure parameters, the phase boundary linear intercept (or indicative mean $\alpha$ plate spacing) and mean $\beta$ phase aspect ratio, were used to assess the microstructure heterogeneity seen in case study examples taken from samples built by an Arcam SEBM machine. The result confirmed that 'lack of fusion' defects were found in 'colder' regions of builds with complex geometry, where the microstructure was shown to be less spheroidised. In comparison, in the centre of the build, the $\alpha-\beta$ transformation microstructure was found to be more uniform across individual layers, although there was evidence of the $\alpha$ lamellar spacing varying systematically with the layer height.

At a larger scale, mapping was performed across entire section widths and the full chamber build height using data acquired from images sampled with a step size of $238.1 \mu \mathrm{m}$. This showed conclusively that there are systematic trends in the morphology of the microstructure, which can be related to the local conditions experienced during building a component.

\section{Acknowledgement}

This project is supported by the LATEST2 program ((EP/G022402/1), Airbus Group Innovations, GKN Aerospace and the EPSRC CDT in Advanced Metallic Systems (EP/L016273/1).

\section{References}

1. L. E. Murr et al., "Metal Fabrication by Additive Manufacturing Using Laser and Electron Beam Melting Technologies," J. Mater. Sci. Technol., 28 (2012), 1-14. 
2. E. Brandl et al., "Additive manufactured Ti-6A1-4V using welding wire: Comparison of laser and arc beam deposition and evaluation with respect to aerospace material specifications," Phys. Procedia, 5 (2010), 595-606.

3. A. A. Antonysamy, "Microstructure, Texture and Mechanical Property Evolution during Additive Manufacturing of Ti6Al4V Alloy for Aerospace Applications" (Ph. D. thesis, University of Manchester, 2012).

4. B. Baufeld, O. V. D. Biest, and R. Gault, "Additive manufacturing of Ti-6Al-4V components by shaped metal deposition: Microstructure and mechanical properties," Mater. Des., 31 (2010), 106-111.

5. S. Leuders et al., "On the mechanical behaviour of titanium alloy TiAl6V4 manufactured by selective laser melting: Fatigue resistance and crack growth performance," International Journal of Fatigue, 48 (2013), 300-307.

6. B. Song, S. Dong, B. Zhang, H. Liao, and C. Coddet, "Effects of processing parameters on microstructure and mechanical property of selective laser melted Ti6Al4V," Mater. Des., 35 (2012), 120-125.

7. S. S. Al-Bermani, M. L. Blackmore, W. Zhang, and I. Todd, "The origin of microstructural diversity, texture, and mechanical properties in electron beam melted Ti-6Al-4V," Metall. Mater. Trans. A Phys. Metall. Mater. Sci., 41 (2010), 3422-3434.

8. A. A. Antonysamy, "The Effect of Integrating Rolling Deformation with Wire Arc Additive Manufacturing of Ti-6Al-4V - on Grain Size and Texture Refinement", Material Characterisation, 84(2013), 153-168.

9. L. Tan and Jean Jiang, Digital Signal Processing (Second Edition) (New York, NY: Elsevier Inc., 2013), 692-718.

10. John W. Woods, Multidimensional Signal, Image and Video Processing and Coding (New York, NY: Elsevier Inc., 2013), 223-255. 